\title{
Pobreza e Distribuição de Renda em Áreas Rurais: uma Abordagem de Inferência
}

Henrique Dantas Neder ${ }^{1}$ e Jorge Luiz Mariano da Silva²

Resumo - O trabalho desenvolve aplicações de metodologias para a estimativa de índices de pobreza e de distribuição de renda em áreas rurais considerando-se os erros amostrais das Pesquisas Amostrais por Amostras Domiciliares - PNADs. São obtidos estimativas com intervalos de confiança para diversos indicadores e para diversas áreas rurais do Brasil e explicitadas as áreas onde se observou alterações significativas na proporção de pobres, intensidade da pobreza e distribuição de renda no período 1995-2001. Os resultados das estimativas apontam para a ocorrência de variação significativa e positiva nos indicadores de pobreza nos Estados de São Paulo, Pernambuco, Alagoas e queda significativa dos mesmos indicadores no Ceará, Rio de Janeiro, Santa Catarina e Goiás. Verificou-se uma queda significativa no mesmo período na concentração de renda nas áreas rurais de Tocantins, Piauí, Paraíba, região metropolitana rural, região Nordeste rural, região Nordeste metropolitana rural e região Sul metropolitana rural e nos estados de São Paulo, Paraná e Ceará, constatou-se uma elevação significativa do valor do índice de Gini. Com base em valores calculados de elasticidades da pobreza foi possível estabelecer uma avaliaçao das regiões rurais do país em termos de efetividade de combate à pobreza em termos de políticas de crescimento e distributivas.

\footnotetext{
${ }^{1}$ Doutor em Economia - Professor da Universidade Federal de Uberlândia, E-mail: dneder@ufu.br.

${ }^{2}$ Doutor em Economia - Professor da Universidade Federal do Rio Grande do Norte
} 
Palavras-chaves: distribuição de renda, pobreza rural, bootstrapping, indicadores sociais

\section{Classificação JEL: I32}

Abstract - The work develops applications of methodologies for the estimation of poverty indexes and income distribution in rural areas, being considered the sample errors of the National Survey for Household Sampling - PNADs. Are obtained estimates with confidence intervals for several indicators and for several rural areas of Brazil and stressed the areas where it was observed significant alterations in the poor proportion, poverty intensity and income distribution in the period 19952001. The results of the estimates point for the occurrence of significant and positive variation in the poverty indicators in States of São Paulo, Pernambuco, Alagoas and significant fall of the same indicators in Ceará, Rio de Janeiro, Santa Catarina and Goiás. A significant fall was verified in the same period in the income concentration of the rural areas of Tocantins, Piaui, Paraíba, rural metropolitan area, rural Northeast area, rural metropolitan Northeast area and rural metropolitan South area and in the states of São Paulo, Paraná and Ceará, a significant elevation of the value of Gini index was verified. With base in calculated values of poverty elasticities was possible to establish an evaluation of the rural areas of the country in terms of combat effectiveness to the poverty based on growth and distributives policies.

Key words: income distribution, rural poverty, bootstrapping, social indicators

\section{JEL Classification: I32}

\section{1 - Introdução}

Com as recentes transformações sociais no Brasil em decorrência do processo de liberalização econômica do país, acompanhado do agravamento da crise social, torna-se relevante o estudo da evolução de indicadores que permitam não apenas apontar as áreas e setores em que 
a pauperização da população é mais dramática e evidente em função da ausência de políticas públicas, como também subsidiar a formulação de políticas eficazes de combate à pobreza e a concentração de renda rural. Também é importante tentar demonstrar que este agravamento poderia ter sido maior se algumas políticas sociais não tivessem sido operantes e efetivas como as decorrentes dos princípios estabelecidos na Constituição Federal de 1988, como é o caso da implementação das aposentadorias rurais e a extensão do direito universal das mesmas para pessoas com mais de 65 anos e sem renda. O argumento de que as políticas sociais foram inócuas em período recente com relação à melhoria dos indicadores, não leva em conta o fato de que o agravamento destes índices teria sido mais dramático caso muitos dos programas não tivessem existido e se mantido na década de 90 . O debate atual sobre as questões sociais no Brasil está hoje centrado na oposição de uma universalização e focalização das políticas sociais. Esta é talvez uma falsa contraposição, na medida em que mesmo advogando-se em favor da manutenção da universalização, existe a necessidade da avaliação dos efeitos das intervenções.

Os efeitos das novas políticas econômicas e sociais nas áreas rurais do Brasil foram bastante diversos e devem ser tratados com atenção particular. Algumas mudanças ocorreram, tal como também para a população urbana, em função da estabilização de preços no início da segunda metade da década de 90, sendo que os efeitos positivos parecem esgotar-se a partir do final deste período, quando as elevadas taxas de desemprego e a situação recessiva do País contrabalançam negativamente os ganhos sociais advindos da queda inflacionária. Algumas transformações econômicas nas áreas rurais parecem ter também contribuído para aplacar os efeitos da crise como é o caso do crescimento das atividades não agrícolas em algumas regiões do Brasil rural. No entanto, verifica-se tal como constatado em Neder/2001 que estas atividades contribuíram para a piora dos índices de distribuição de renda rural. Um importante componente da renda rural, que são as aposentadorias, passou a ter um papel relevante como fator distributivo de renda. ${ }^{3}$ È provável que a forma tal como se direciona a trajetória

${ }^{3}$ Em recente trabalho, Hoffmann (2003) demonstra, através do uso de técnica de decomposição do índice de Gini, que as aposentadorias de uma forma geral são 
do desenvolvimento rural seja tão ou mais perversa do que a chamada modernização conservadora, tendo em vista as elevadas taxas de desemprego rural em virtude da intensificação da mecanização das atividades agrícolas, como também de uma certa extensão do novo paradigma tecnológico e de organização produtiva para as áreas rurais.

\section{2 - Metodologia}

\section{1 - Considerações sobre o tratamento das amostras utilizadas}

O presente trabalho baseia-se na estimativa de índices de pobreza e de concentração de renda obtidos das PNADs - Pesquisa Nacional por Amostras Domiciliares em dois anos: 1995 e 2001. O cálculo dos índices foi realizado utilizando-se procedimentos de inferência que além de obter as estimativas de ponto foram também estimados intervalos de confiança para os indicadores tendo em conta que os dados provém de amostras domiciliares. A PNAD é uma pesquisa anual com desenho de amostragem complexa e que necessita de um tratamento de inferência que leve em conta estas características. Nas regiões metropolitanas e nos chamados municípios auto-representativos (municípios de grande população situados fora das regiões metropolitanas) a amostra é realizada em dois estágios sendo que a unidade primária amostral (PSU) são os setores censitários. Estes são selecionados com probabilidade proporcional ao tamanho (PPS) sendo utilizado como proxy de tamanho o número de domicílios em cada setor. Em cada setor selecionado são escolhidos domicílios através de um procedimento de amostragem sistemática. Existe também uma estratificação implícita feita pelo IBGE a partir do ordenamento dos setores censitários para a seleção dos setores da amostra. Para os municípios não auto-representativos (municípios pequenos), as unidades da federação são estratificadas geograficamente, sendo que em cada estrato são selecionados dois municípios, sendo estes as unidades primárias de amostragem (PSUs). A seleção dos municípios

concentradoras de renda. Por outro lado, Neder (2001) verifica que as aposentadorias rurais têm efeito desconcentrador sobre a distribuição de renda rural. Ver também Delgado e Cardoso (2000) para uma análise do efeito da universalização de direitos sociais sobre as condições de vida da população rural. 
é feita com probabilidade proporcional ao tamanho dos mesmos, sendo que a proxy de tamanho indicada é a população total do município por ocasião do último censo. Após esta etapa são selecionados os setores censitários adotando-se o mesmo método empregado nas regiões metropolitanas e municípios auto-representativos. Desta forma, para os municípios não auto-representativos a amostra é em três estágios. ${ }^{4}$

O que importa para fins de utilização das informações do desenho de amostragem para realizar inferências é conhecer em qual estrato e em qual PSU está localizado o domicílio da amostra. Com este conhecimento e de posse dos registros unitários (micro-dados) das PNADs, assim como programas estatísticos convenientes, é possível não somente estimar índices, assim como obter a dimensão dos erros de amostragem para cada estimativa. Deve-se levar em conta que os erros de amostragem em uma amostra complexa como é o caso da PNAD são muito mais elevados do que os mesmos erros para uma amostra aleatória simples (AAS) com o mesmo tamanho de amostra. A variância dos estimadores amplifica-se em relação à mesma variância de uma AAS na medida do chamado fator do desenho da amostra que irá depender das características descritas anteriormente.

Cabe um comentário sobre a comparabilidade entre os dados das amostras da PNAD 1995 e 2001. Como neste artigo foram consideradas as sub-amostras para as áreas rurais do país e levando-se em conta que para o ano de 2001 a PNAD redefine as áreas rurais de acordo com os resultados do Censo Demográfico de 2001, é possível que ocorra alguma perda de comparabilidade entre as estimativas para os dois anos. O que ocorre é que as estimativas para 2001 por estarem mais próximas do ano censitário do que as estimativas para 1995, elas acabem por refletir com maior rigor os valores dos indicadores para o conjunto das áreas rurais do país e nas regiões estudadas. Esta distorção seria mais grave quanto mais afastado estivesse o ano inicial (1995) estivesse do ano censitário correspondente (1991). É provável que em quatro anos o perímetro urbano de muitos municípios tenha se ampliado, mas desprezaremos aqui este

\footnotetext{
${ }^{4}$ Para uma compreensão mais detalhada dos fundamentos teóricos de uma amostragem complexa tal como a amostra da PNAD, ver Pessoa e Silva (1998). Uma descrição clara do delineamento amostral da PNAD pode ser encontrada em Silva (2002).
} 
efeito levando-se em conta que muitas vezes esta ampliação não reflete necessariamente o processo de urbanização no inicio da década de 1990, sendo decorrência muitas vezes dos interesses das municipalidades em ampliar a arrecadação de impostos via cobrança de IPTU.

\section{2 - Métodos de estimativa de intervalos de confiança para os indicadores}

Neste trabalho serão empregados para efeito de checagem diversos métodos de estimativa. Um primeiro método é o da estimativa da variância baseado na "linearização" (Stata, 2001 e Pessoa e Silva, 2002) empregando os comandos svy do Stata. Para as estimativas dos índices de pobreza foi utilizado um programa do Stata (ado file) denominado sepov (Jollife e Semykina,1999). Este programa estima os índices de Foster-Greer-Thorbecke (headcount ratio $-\mathrm{P}_{0}$, poverty gap $-\mathrm{P}_{1} e$ squared poverty gap $-\mathrm{P}_{2}$ ) com as suas respectivas variâncias e limites de intervalo de confiança. Estes índices são calculados com base nas seguintes expressões:

$$
\begin{aligned}
& P_{0}=\frac{q}{n} \\
& P_{1}=\frac{1}{n} \sum_{i=1}^{q} \frac{z-y_{i}}{z} \\
& P_{2}=\frac{1}{n} \sum_{i=1}^{q}\left(\frac{z-y_{i}}{z}\right)^{2}
\end{aligned}
$$

onde q é o número de pobres (pessoas cuja renda per capita domiciliar é menor que a linha de pobreza), n é o tamanho da população, z é a linha de pobreza e $y_{i}$ é a renda per capita domiciliar da i-ésima pessoa.

O primeiro índice (Poverty Headcount) mede a proporção de pessoas pobres, ou seja, a proporção de pessoas que têm renda per capita domiciliar inferior à linha de pobreza. Tal indicador é utilizado como ponto de partida para estudos sobre pobreza, mas é insuficiente para analisá-la dado que viola dois importantes axiomas (Sen apud Prates, 1996). Em primeiro lugar, o indicador não se altera ao se reduzir a renda de uma pessoa situada abaixo da linha de pobreza ou o inverso, isto é, quando a renda eleva-se sem alcançar a linha de pobreza o indicador permanece o mesmo. Em segundo lugar, a proporção também é insensível 
à distribuição de renda entre os pobres, não se alterando quando se transfere renda de um indivíduo mais pobre para outro menos pobre. Sendo assim, a proporção de pobres deve ser utilizada em conjunto com outros dois indicadores, que se complementam mutuamente.

O segundo índice ("poverty gap") mede a intensidade de pobreza para o conjunto da população pobre através do cálculo do desvio médio entre a renda dos pobres e o valor da linha de pobreza e pode ser interpretado como um indicador do déficit de pobreza, ou seja, os recursos necessários para elevar a renda de todos os pobres ao nível da linha de pobreza, através de uma perfeita focalização das transferências de renda. O terceiro índice (Squared Poverty Gap) é geralmente descrito como um indicador de severidade da pobreza. Na construção deste índice utiliza-se um peso maior para as pessoas mais pobres (o "gap de pobreza” é ponderado por si mesmo) e leva-se em conta a desigualdade de renda entre os pobres. Considerando-se a utilização destes índices para os propósitos de políticas públicas de combate à pobreza a proporção de pobres $\left(\mathrm{P}_{0}\right)$ atribui maior efetividade à políticas que elevam a renda dos menos pobres (aqueles cuja renda é mais próxima de $\mathrm{z}$ ). Já o gap de pobreza $\left(\mathrm{P}_{1}\right)$ e o gap de pobreza ao quadrado $\left(\mathrm{P}_{2}\right)$ colocam ênfase naqueles que estão muito abaixo de $z$, ou seja, os mais pobres dos pobres. As linhas de pobreza utilizadas foram obtidas nos trabalhos da pesquisadora do Instituto de Pesquisas Econômicas Aplicadas - IPEA, Sonia Rocha, e cuja metodologia encontra-se descrita em Rocha(1998).

Um segundo método empregado para a estimativa dos indicadores é o bootstrapping. Este método baseia-se em procedimentos de reamostragem (replicação de inúmeras amostras selecionadas com reposição da amostra original e com mesmo tamanho n). Foram obtidas para cada estimativa 100 replicações e feito o cálculo do desvio-padrão dos indicadores a partir da distribuição do índice nas replicações. Este método é particularmente adequado para a inferência dos índices de distribuição, particularmente o índice de Gini, na medida que este tem uma fórmula complexa e para o qual não pode ser adotado um método analítico (como o primeiro método). O método do bootstrapping para uma amostra complexa procura imitar, para a construção dos replicates (amostras selecionadas com reposição selecionadas da amostra original), o mesmo método de seleção adotado para a seleção da amostra da PNAD. 
Assim, da própria amostra original da PNAD são selecionados os PSUs com reposição com probabilidade proporcional ao tamanho. Para os PSUs de regiões metropolitanas e municípios auto-representativos foi utilizada a probabilidade de seleção do município como variável psuwt e para os municípios não auto-representativos foi utilizada a probabilidade de seleção do setor censitário, sendo que ambas variáveis estão disponíveis nos arquivos de micro-dados das PNADs. As estimativas dos intervalos de confiança basearam-se na hipótese de normalidade da distribuição amostral (para maiores detalhes ver Mooney e Duval (1993) assim como Stata (2001)).

Um terceiro método de estimativa deintervalos de confiança empregado neste trabalho é a abordagem de linearização de Rao. Este método é descrito com detalhes no manual do programa DAD da Universidade de Laval (Duclos et alii, 2002) e foi utilizado no contexto das estimativas de intervalos de confiança dos índices de Gini sendo comparados com as mesmas estimativas obtidas pelo método bootstrapping através do programa Stata. Os resultados da comparação comprovaram-se satisfatórios sendo as variâncias obtidas muito próximas.

\section{3 - Cálculo da elasticidade da pobreza}

Para o cálculo da elasticidade da pobreza foi utilizada a metodologia proposta por Datt (1998). Esta é uma proposição para cálculo dos valores das elasticidades a partir de dados de distribuição de renda agrupados. O autor do presente artigo desenvolveu um programa em Stata adaptando a metodologia para a estrutura dos micro-dados das PNADs. No artigo de Datt (1998) são apresentadas duas especificações para a curva de Lorenz, mas optamos por simplicidade apenas para a Quadrática Geral. ${ }^{5}$ Sejam as seguintes funções:

$$
\begin{aligned}
& \text { Curva de Lorenz: } L=L(p ; \pi) \\
& \text { e } \\
& \text { Medida de pobreza: } P=P(\mu / z ; \pi)
\end{aligned}
$$

\footnotetext{
${ }^{5}$ Pretende-se em um próximo trabalho estimar os parâmetros da curva de Lorenz utilizando também a especificação Beta (mostrada em Datt, 1998) e verificar qual
} 
onde $\mathrm{L}$ é a participação dos p por cento inferiores da população na renda per capita domiciliar, $\pi$ é um vetor de parâmetros estimáveis da curva de Lorenz, P é a medida de pobreza escrita como uma função da relação da renda per capita domiciliar média $\mu$ à linha de pobreza $z$ e os parâmetros da curva de Lorenz. A especificação Quadrática Geral da curva de Lorenz é dada pelas seguintes funções:

$$
\begin{aligned}
& L(1-L)=a\left(p^{2}-L\right)+b L(p-1)+(c-L) \\
& \text { ou } \\
& L(1-L)=-\frac{1}{2 m}\left[b p+e+\left(m p^{2}+n p+e^{2}\right)^{1 / 2}\right]
\end{aligned}
$$

As funções que representam as medidas de pobreza dadas a partir desta especificação da curva de Lorenz são:

$$
\begin{aligned}
& H=-\frac{1}{2 m}\left[n+r(b+2 z / m)\left\{(b+2 z / m)^{2}-m\right\}^{-1 / 2}\right] \\
& P G=H-(m / z) L(H) \\
& P_{2}=2(P G)-H-\left(\frac{\mu}{z}\right)^{2}\left[a H+b L(H)-\left(\frac{r}{16}\right) \ln \left(\frac{1-H / s_{1}}{1-H / s_{2}}\right)\right]_{(8)}
\end{aligned}
$$

onde $\mathrm{H}, \mathrm{PG}$ e $\mathrm{P}_{2}$ são respectivamente a proporção de pobres, o gap de pobreza e a severidade da pobreza da classe dos índices FGT de pobreza e os parâmetros:

$$
\begin{aligned}
& e=-(a+b+c+1) \\
& m=b^{2}-4 a \\
& n=2 b e-4 c \\
& r=\left(n^{2}-4 m e^{2}\right)^{1 / 2} \\
& s_{1}=(r-n) /(2 m) \\
& s_{2}=-(r+n) /(2 m)
\end{aligned}
$$

das duas (para cada conjunto de dados amostrais) cumpre mais adequadamente as condições de fronteira e de monotonicidade. Existem também métodos de estimativa e de análise diversos para a elasticidade da pobreza em relação ao crescimento, apresentados em Heltberg (2002). 
O procedimento para estimar os parâmetros da curva de Lorenz utilizou uma regressão linear por mínimos quadrados ordinários de L(1L) em $\left(p^{2}-L\right), L(p-1)$ e (p-L) para a obtenção dos parâmetros a, b e c.

Os valores das elasticidades da pobreza são calculadas a partir das fórmulas no Quadro 1, a seguir:

Quadro 1 - Fórmulas da elasticidade da pobreza em relação à renda média e ao índice de Gini

\begin{tabular}{|l|l|c|}
\hline \multicolumn{1}{|c|}{ Elasticidade da } & Média 0 & Índice de Gini \\
\hline $\mathrm{H}$ & $-z /\left(\mu H L^{\prime \prime}(H)\right)$ & $(1-z / \mu) /\left(H L^{\prime \prime}(H)\right)$ \\
\hline PG & $1-H / P G$ & $1+(\mu / z-1) H / P G$ \\
\hline SPG & $2\left(1-P G / P_{2}\right)$ & $2\left[1+(\mu / z-1) P G / P_{2}\right]$ \\
\hline
\end{tabular}

Fonte: Fórmulas obtidas de Datt (1998) . O valor de L'(H) é o valor da segunda derivada da curva de Lorenz e igual a $\frac{r^{2}\left(m H^{2}+n p+e^{2}\right)^{-3 / 2}}{8}$

\section{3 - Resultados}

Na Tabela 1 são apresentadas as estimativas da proporção de pobres $\left(\mathrm{P}_{0}\right)$ para os anos de 1995 e 2001 com os respectivos intervalos de confiança e níveis de significância dos valores da diferença entre os índices de 2001 e 1995. Nos Estados do Ceará, Rio de Janeiro, Santa Catarina e Goiás observa-se uma queda significativa na proporção de pobres $\left(\mathrm{P}_{0}\right)$, com níveis de significância 0,0973; 0,0251;0,0626 e 0,0580 respectivamente. ${ }^{6}$ No Estado de São Paulo rural observa-se uma elevação significativa da proporção de pobres entre os anos de 1995 e 2001, com nível de significância 0,0366. Na Tabela 2 são apresentados os valores dos hiatos de pobreza $\left(\mathrm{P}_{1}\right)$ para os mesmos anos ocorrendo uma queda significativa nos Estados do Piauí, Paraíba, Santa Catarina e Goiás e elevação significativa deste índice nos Estados de Pernambuco, Alagoas e São Paulo. Portanto os Estados que tiveram redução significativa da pobreza tanto no que se refere a sua proporção como a sua intensidade foram Santa Catarina e Goiás enquanto que em São Paulo observou-se um agravamento nestes dois aspectos da pobreza. Os dados da Tabela

\footnotetext{
${ }^{6}$ Para a interpretação dos “p-values”, ou níveis de significância, considera-se que estes são as probabilidades de ocorrência de um erro tipo I (rejeitar a hipótese nula - variação nula do índice, quando esta hipótese é verdadeira). No caso do Estado do Ceará o valor do p-value é 0,0973 significando que ao rejeitar a hipótese nula estamos incorrendo em uma probabilidade de erro de 9,73 \%, o que é aceitável. Quanto menor o valor do p-value com mais confiança podemos afirmar que houve uma variação significativa do índice.
} 
3 são mais agregados por regiões e mostram uma queda significativa da proporção de pobres no período 1995-2001 na região Centro-Oeste rural, na região rural do estado do Rio de Janeiro, ao passo que se observa um aumento significativo da intensidade da pobreza (índice $\mathrm{P}_{1}$ ) além de em São Paulo, como visto anteriormente, mas também na região Nordeste 2 formada pelos Estados de Alagoas, Pernambuco, Sergipe e Bahia. Na Tabela 3, a região Nordeste foi dividida em duas sub-regiões (NE1 rural e NE2 rural). Procedeu-se dessa forma para que fosse possível aproveitar melhor as possibilidades de desagregação das amostras, levando-se em conta que a sub-amostra para o Nordeste rural é muito grande em relação às demais regiões do país, devido ao seu elevado número de unidades da federação.

Tabela 1 - Proporção de pobres em áreas rurais por unidade da federação 1995 e 2001

\begin{tabular}{l|c|c|c|c|c|c|c}
\hline & \multicolumn{2}{|c|}{$\underline{\mathbf{1 9 9 5}}$} & \multicolumn{2}{c|}{$\underline{\mathbf{2 0 0 1}}$} & \multicolumn{2}{c|}{ Diferença } & \\
\hline \multicolumn{1}{c|}{ Área } & estimativa & variância & estimativa & variância & estimativa & Variância & p-value \\
\hline CE & 0,5967 & 0,0003 & 0,5460 & 0,0012 & $-0,0506$ & 0,00150 & 0,0973 \\
\hline RJ & 0,3364 & 0,0010 & 0,2498 & 0,0010 & $-0,0866$ & 0,0020 & 0,0251 \\
\hline SP & 0,1816 & 0,0004 & 0,2340 & 0,0005 & 0,0524 & 0,0009 & 0,0366 \\
\hline SC & 0,2044 & 0,0006 & 0,1430 & 0,0010 & $-0,0614$ & 0,0016 & 0,0626 \\
\hline GO & 0,4028 & 0,0013 & 0,3249 & 0,0012 & $-0,0779$ & 0,0025 & 0,0580 \\
\hline
\end{tabular}

Fonte dos dados básicos: microdados da PNAD - FIBGE

Nota: Estimativas obtidas pelo método da linearização de Taylor (programa SEPOV do Stata). São apresentados na tabela apenas os resultados com p-value menor do que 0,10.

Tabela 2 - Hiato de pobreza em áreas rurais por unidade da federação 1995 e 2001

\begin{tabular}{l|c|c|c|c|c|c|c}
\hline & \multicolumn{2}{|c|}{$\mathbf{1 9 9 5}$} & \multicolumn{2}{c|}{2001} & \multicolumn{2}{c|}{ Diferença } & \\
\hline Área & estimativa & variância & estimativa & variância & estimativa & Variância & p-value \\
\hline PI & 0,3581 & 0,0012 & 0,2960 & 0,0006 & $-0,0621$ & 0,0019 & 0,0745 \\
\hline PB & 0,2891 & 0,0009 & 0,2324 & 0,0006 & $-0,0567$ & 0,0015 & 0,0729 \\
\hline PE & 0,2188 & 0,0005 & 0,2556 & 0,0001 & 0,0368 & 0,0006 & 0,0652 \\
\hline AL & 0,1987 & 0,0004 & 0,2577 & 0,0008 & 0,0591 & 0,0012 & 0,0434 \\
\hline SP & 0,0696 & 0,0001 & 0,1126 & 0,0002 & 0,0430 & 0,0002 & 0,0019 \\
\hline SC & 0,0886 & 0,0002 & 0,0575 & 0,0001 & $-0,0311$ & 0,0003 & 0,0317 \\
\hline GO & 0,1735 & 0,0006 & 0,1305 & 0,0004 & $-0,0429$ & 0,0010 & 0,0870 \\
\hline
\end{tabular}

Fonte dos dados básicos: micro-dados da PNAD - FIBGE

Nota: Estimativas obtidas pelo método da linearização de Taylor (programa SEPOV do Stata). São apresentados na tabela apenas os resultados com p-value menor do que 0,10 . 
Tabela 3 - Proporção de pobres e hiato de pobreza por regiões - 1995 e 2001

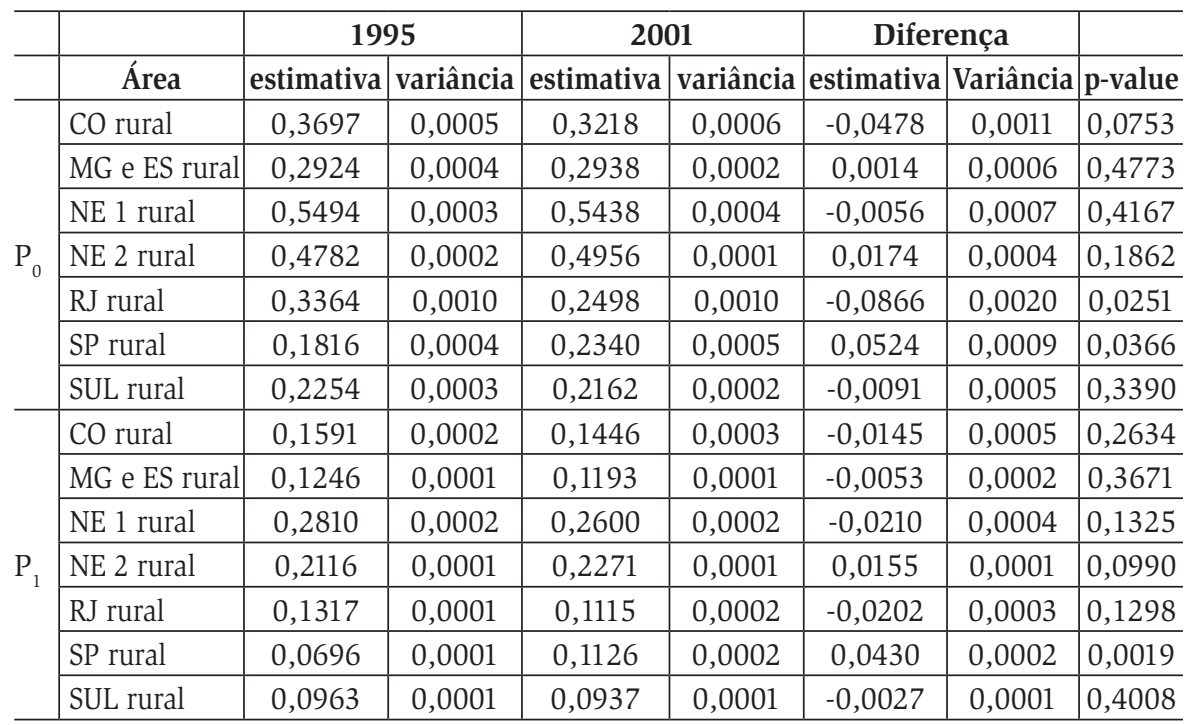

Fonte dos dados básicos: micro-dados da PNAD - FIBGE

Nota: NE 1 (MA,PI,CE,RN,PB) e NE 2 (PE,AL,SE,BA)

Nota: Estimativas obtidas pelo método da linearização de Taylor (programa SEPOV do Stata).

A Tabela 4 apresenta os valores dos índices de Gini assim como também os seus respectivos intervalos de confiança para os anos de 1995 e 2001 para as diversas áreas rurais do Brasil. Nesta tabela também são mostradas as diferenças dos valores dos índices para estes dois anos e o valor do nível de significância ( $p$-value) para estas diferenças. Observase uma queda significativa do índice de Gini (valores negativos para a diferença e p-value baixo) nos Estados do Tocantins, Piauí, Paraíba e nas regiões rurais metropolitanas, no Nordeste rural como um todo, no Nordeste rural metropolitano e na região Sul rural metropolitana. Constatam-se elevações significativas do índice de Gini (valores positivos da diferença e p-value baixo) nas áreas rurais dos Estados de São Paulo, Ceará e Paraná. No conjunto das áreas rurais do Brasil não é observada uma variação significativa do índice de Gini ( $p$-value $=0,4056)$ no período 1995-2001. 
Tabela 4 - Índices de Gini com intervalos de confiança e teste de hipótese para a diferença dos índices - 1995 e 2001, áreas rurais

\begin{tabular}{l|c|c|c|c|c|c|c|c|c|c|c}
\hline \multicolumn{1}{c|}{ Área } & gini1995 & desvio & linf & lsup & gini2001 & desvio & linf & lsup & diferença & desvio & p-value \\
\hline TO & 0,6997 & 0,0576 & 0,5856 & 0,8142 & 0,5206 & 0,0329 & 0,4553 & 0,5859 & $-0,1791$ & 0,0663 & 0,0035 \\
\hline PI & 0,5809 & 0,0240 & 0,5332 & 0,6286 & 0,5218 & 0,0168 & 0,4885 & 0,5552 & $-0,0591$ & 0,0293 & 0,0218 \\
\hline CE & 0,4665 & 0,0142 & 0,4384 & 0,4946 & 0,5144 & 0,0135 & 0,4876 & 0,5413 & 0,0479 & 0,0196 & 0,0072 \\
\hline PB & 0,5232 & 0,0356 & 0,4524 & 0,5939 & 0,4281 & 0,0133 & 0,4017 & 0,4546 & $-0,0951$ & 0,0380 & 0,0062 \\
\hline SP & 0,4713 & 0,0190 & 0,4336 & 0,5090 & 0,5172 & 0,0221 & 0,4734 & 0,5610 & 0,0459 & 0,0291 & 0,0576 \\
\hline PR & 0,5103 & 0,0236 & 0,4634 & 0,5572 & 0,5579 & 0,0285 & 0,5015 & 0,6144 & 0,0476 & 0,0370 & 0,0992 \\
\hline $\begin{array}{l}\text { Brasil- } \\
\text { Metro }\end{array}$ & 0,5367 & 0,0278 & 0,4814 & 0,5919 & 0,4602 & 0,0149 & 0,4307 & 0,4897 & $-0,0765$ & 0,0315 & 0,0076 \\
\hline Nordeste & 0,5274 & 0,0101 & 0,5073 & 0,5475 & 0,5042 & 0,0142 & 0,4762 & 0,5323 & $-0,0232$ & 0,0174 & 0,0915 \\
\hline $\begin{array}{l}\text { Nordeste- } \\
\text { Metr }\end{array}$ & 0,6099 & 0,1149 & 0,3820 & 0,8378 & 0,4396 & 0,0397 & 0,3608 & 0,5184 & $-0,1703$ & 0,1216 & 0,0806 \\
\hline Sul- Metr & 0,5186 & 0,0297 & 0,4597 & 0,5774 & 0,4496 & 0,0159 & 0,4180 & 0,4813 & $-0,0690$ & 0,0337 & 0,0203 \\
\hline
\end{tabular}

Fonte dos dados básicos: microdados da PNAD - FIBGE

Nota: Estimativas obtidas pelo método do bootstrapping através do programa ineqerr (adaptado) do Stata.

São apresentados na tabela apenas os resultados com p-value menor do que 0,10.

A Tabela 5 apresenta as elasticidades da pobreza com relação ao aumento da renda média e do índice de Gini considerando-se os três índices FGT. As elasticidades representam a variação percentual no índice de pobreza como decorrência de uma variação percentual unitária da renda média (elasticidade da pobreza em relação ao crescimento) e a variação percentual no índice de pobreza como decorrência de uma variação percentual unitária no índice de Gini (elasticidade da pobreza em relação ao índice de Gini). Nesta Tabela são também apresentados os valores estimados dos índices de pobreza e Gini utilizando-se os métodos descritos na seção 2.3 e observa-se uma boa aproximação destes valores aos obtidos através dos outros métodos. Considerandose a elasticidade da pobreza (índice $P_{0}$ ) em relação ao crescimento da renda média, os Estados que apresentaram em 2001 os melhores resultados são Espírito Santo, Rio de Janeiro, Santa Catarina, Mato Grosso do Sul e São Paulo ao passo que o Piauí, Maranhão, Ceará, Paraíba e a região Nordeste rural mostraram índices mais reduzidos em valores absolutos. A interpretação desses resultados, por exemplo para os estados do Piauí e Espírito Santo, que registraram as elasticidades da pobreza de $-0,7326$ e $-1,3075$, respectivamente, indicam, que se 
houver um acréscimo de $1 \%$ na renda média dos domicílios nas regiões rurais nesses estados, o percentual de pobres cairia no Piauí em 0,73\%, enquanto no Espírito Santo em 1,3\%.

Tabela 5 - Elasticidades da pobreza em relação ao crescimento da renda média e ao índice de Gini - áreas rurais 2001

\begin{tabular}{|c|c|c|c|c|c|c|c|c|c|c|}
\hline Área & elcresP0 & elcresP1 & elcresP2 & elginiP0 & elginiP1 & elginip2 & gini & P0 & P1 & P2 \\
\hline TO & $-0,9309$ & $-1,0972$ & 1,2015 & 0,6893 & 2,5528 & 4,3703 & 0,5101 & 0,4541 & 0,2165 & 0,1353 \\
\hline $\mathrm{NE}$ & 569 & 0724 & 2064 & 058 & 3 & 3,4811 & 38 & 82 & 2501 & 1560 \\
\hline MA & $-0,7398$ & $-1,1067$ & $-1,3915$ & 0,5781 & 2,6463 & 4,6503 & 0,3524 & 0,5426 & 0,2576 & 0,1519 \\
\hline PI & 0,7326 & $-0,8943$ & $-0,9614$ & 0,1993 & 1,5154 & 2,8058 & 0,5215 & 0,5795 & 0,3059 & 0,2066 \\
\hline CE & $-0,7519$ & $-0,9327$ & $-1,0289$ & 0,2657 & 1,6828 & 3,0701 & 0,5138 & 0,5524 & 0,2858 & 0,1887 \\
\hline $\mathrm{RN}$ & $-0,9353$ & $-1,0668$ & $-1,1407$ & 0,7636 & 2,6873 & 4,5641 & 0,4901 & 0,4431 & 0,2144 & 0,1365 \\
\hline $\mathrm{PE}$ & $-0,9670$ & $-1,1913$ & $-1,3313$ & 0,2742 & 1,6214 & 2,9447 & 0,4281 & 0,5102 & 0,2328 & 0,1398 \\
\hline $\mathrm{PB}$ & $-0,8492$ & $-1,0362$ & $-1,1367$ & 0,2651 & 1,6356 & 2,9791 & 0,4749 & 0,5341 & 0,2623 & 0,1673 \\
\hline $\mathrm{AL}$ & $-0,8706$ & $-1,0785$ & $-1,1979$ & 0,2458 & 1,5868 & 2,9028 & 0,4598 & 0,5348 & 0,2573 & 0,1609 \\
\hline SE & $-0,9516$ & $-1,2936$ & $-1,5633$ & 0,4438 & 2,0696 & 3,6617 & 0,4411 & 0,4761 & 0,2076 & 0,1165 \\
\hline $\mathrm{BA}$ & $-0,9423$ & $-1,1727$ & $-1,3290$ & 503 & 14 & 3 & 0,4731 & 0,4846 & 0,2230 & 0,1340 \\
\hline MG e ES & $-1,0112$ & $-1,3153$ & $-1,5765$ & 2,1154 & 5,8438 & 9,4822 & 0,4191 & 0,3316 & 0,1432 & 0,0801 \\
\hline MG & $-1,1547$ & $-1,2393$ & $-1,3112$ & 1,8444 & 4,5768 & 7,2891 & 0,4373 & 0,2887 & 0,1289 & 0,0779 \\
\hline ES & $-1,30$ & 37 & 4 & 7 & 04 & 46 & 0,3838 & 0,2959 & 0,1069 & 0509 \\
\hline RJ & $-1,3007$ & \begin{tabular}{|l|}
$-1,3772$ \\
\end{tabular} & $-1,4558$ & 1,8504 & 4,3820 & 6,9165 & 0,4153 & 0,2658 & 0,1118 & 0,0647 \\
\hline SP & $-1,2055$ & $-1,2$ & $-1,2834$ & 222 & 39 & 8 & 59 & 79 & 49 & 700 \\
\hline SU & $-1,1067$ & $-1,1463$ & $-1,1840$ & 2,6619 & 6,1623 & 9,6583 & 0,5069 & 0,2133 & 0,0994 & 0,0624 \\
\hline PR & $-1,0772$ & $-1,2659$ & $-1,4300$ & 2,3224 & 5,8849 & 9,3947 & 0,4838 & 0,2787 & 0,1230 & 0,0717 \\
\hline $\mathrm{SC}$ & $-1,2606$ & $-1,2764$ & $-1,3152$ & 3,3628 & 7,0722 & 10,8433 & 0,4414 & 0,1405 & 0,0617 & 0,0372 \\
\hline RS & $-1,0775$ & $-1,0534$ & $-1,0324$ & 2,6801 & 6,1075 & 9,5427 & 0,4920 & 0,1996 & 0,0972 & 0,0641 \\
\hline $\mathrm{CO}$ & $-1,1155$ & $-1,2502$ & $-1,3575$ & 1,5105 & 4,0592 & 45 & 0,4330 & 0,3354 & 0,1491 & 0,0888 \\
\hline MS & $-1,2118$ & $-1,2880$ & $-1,3550$ & 1,7402 & 4,2857 & 6,8179 & 0,4198 & 0,2924 & 0,1278 & 0,0762 \\
\hline MT & $-1,0460$ & $-0,9686$ & $-0,8706$ & 1,0640 & 3,0024 & 4,9200 & 0,4957 & 85 & 0,1770 & 0,1233 \\
\hline GO & $-1,1951$ & $-1,5436$ & $-1,8513$ & 1,6535 & 4,5194 & 7,3287 & 0,3863 & 0,3495 & 0,1374 & 0,0714 \\
\hline DF & $-1,0084$ & $-1,3209$ & $-1,5947$ & 3,0853 & 8,1012 & 12,9987 & 0,6043 & 0,2773 & 0,1195 & 0,0665 \\
\hline
\end{tabular}

Fonte dos dados básicos: micro-dados da PNAD.

Considerando-se o Estado do Espírito Santo cuja estimativa de número de pessoas pobres residentes em áreas rurais em 2001 era de 
153.772, o aumento de $1 \%$ na renda de todas as famílias implicará em uma redução de 2.011 pessoas pobres, ao passo que no Estado do Piauí para o qual era estimado um total de 594.022 pessoas pobres, o mesmo aumento geral de $1 \%$ na renda das famílias possibilitaria uma redução de 4.352 pessoas pobres. É importante destacar que em termos relativos à população pobre original o efeito desta redução para o Estado do Espírito Santo é mais forte tendo em vista o seu maior valor absoluto da elasticidade da pobreza. Se antes da elevação da renda os recursos necessários para erradicar completamente a pobreza no Estado do Espírito Santo seriam $\mathrm{R} \$ 2.792 .396$, passam a ser iguais a $\mathrm{R} \$ 2.743 .007$ após a elevação, implicando em uma redução de R\$ 49.389 mensais. No Estado do Piauí, o mesmo montante antes da elevação da renda seria de $\mathrm{R}$ \$ 16.450.467 e passaria a ser $\mathrm{R}$ \$ 16.303.351 após a elevação, implicando em uma redução de $\mathrm{R} \$ 147.117$ mensais necessários para a erradicação completa da pobreza rural (ver Tabela 6).

De acordo com as conclusões do artigo de Heltberg (2002) a partir de diversos estudos econométricos, a elasticidade da pobreza cresce monotonicamente com o crescimento da renda média e isto explicaria a posição mais vantajosa dos Estados mais desenvolvidos de acordo com os resultados anteriores. Heltberg (op.cit.) também enfatiza que a elasticidade da pobreza depende fortemente do grau de desigualdade: "uma distribuição desigual é um impedimento sério ao efetivo alívio da pobreza", o que torna de certa forma a dicotomia "crescimento versus redistribuição" um falso dilema. Resultados semelhantes são encontrados nos trabalhos de Ravallion e Datt (1994), Bell e Rich (1994) e Shujie, 1999. Esses autores estimaram a elasticidade de várias medidas de pobreza - a proporções de pobres, o hiato de pobreza e o quadrado do hiato de pobreza - e encontraram que essas elasticidades são mais altas quanto maior o impacto do crescimento da renda sobre a renda média dos mais pobres.

Para o Estado do Piauí rural o aumento percentual unitário do índice de Gini ocasionaria uma elevação no índice $P_{0}$ de $0,20 \%$, enquanto que para o Estado de Santa Catarina esta elevação seria de 3,36 \% . Considerando-se um efeito simétrico da alteração do índice de Gini, no último Estado uma política redistributiva seria mais efetiva. 
Tabela 6 - Efeitos da variação de $1 \%$ na renda média e no índice de Gini - Estados do Espírito Santo e Piauí - áreas rurais - 2001

\begin{tabular}{l|c|c|c|c|c|c|c|c}
\hline \multicolumn{1}{c}{ Efeitos do crescimento } \\
\hline UF & LP (R\$) & $\mathbf{n}$ & $\mathbf{n p 1}$ & $\mathbf{n p 2}$ & dif & $\begin{array}{c}\text { R1 (R\$ } \\
\text { /mês) }\end{array}$ & $\begin{array}{c}\text { R2 (R\$/ } \\
\text { mês) }\end{array}$ & $\begin{array}{c}\text { Dif (R\$ / } \\
\text { mês) }\end{array}$ \\
\hline ES & 50,188 & 566588 & 153772 & 151761 & 2011 & 2792396 & 2743007 & 49389 \\
\hline PI & 53,862 & 1031826 & 594022 & 589670 & 4352 & 16450467 & 16303351 & 147117 \\
\hline \multicolumn{7}{c}{ Efeitos da distribuição de renda } \\
\hline UF & LP (R\$) & $\mathbf{n}$ & $\mathbf{n p 1}$ & $\mathbf{n p 2}$ & dif & $\begin{array}{c}\text { R1 (R\$ } \\
/ \mathbf{m e ̂ s})\end{array}$ & $\begin{array}{c}\text { R2 (R\$/ } \\
\text { mês) }\end{array}$ & $\begin{array}{c}\text { Dif (R\$ / } \\
\text { mês) }\end{array}$ \\
\hline ES & 50,188 & 566588 & 153772 & 150200 & 3572 & 2792396 & 2627131 & 165265 \\
\hline PI & 53,862 & 1031826 & 594022 & 592838 & 1184 & 16450467 & 16201177 & 249290 \\
\hline
\end{tabular}

Legenda: $\mathrm{LP}=$ linha de pobreza adotada; $\mathrm{n}=$ total de pessoas; $\mathrm{np} 1=$ número de pessoas pobres antes da alteração (renda média ou índice de Gini); $\mathrm{np} 2$ = número de pessoas pobres após a alteração; dif = diferença, R1 = recursos mensais necessários elevar a renda de todas as pessoas pobres à linha de pobreza antes da alteração; R2 = mesmos recursos necessários após a alteração.

\section{4 - Conclusões}

A análise das estimativas dos indicadores de pobreza para as áreas rurais indicou uma queda significativa na proporção de pobres nos Estados do Ceará, Rio de Janeiro, Santa Catarina e Goiás no período 19952001, enquanto que no Estado de São Paulo observou-se uma elevação significativa dos mesmos. Quanto ao índice de Gini foi constatada uma redução significativa no mesmo período para os Estados do Tocantins, Piauí, Paraíba e nas regiões metropolitanas rurais, no Nordeste rural como um todo, no Nordeste rural metropolitano e na região Sul rural metropolitana. Foram também observadas elevações significativas do índice de Gini nas áreas rurais dos Estados de São Paulo e Paraná. Em termos de distribuição de renda rural a região Nordeste apresenta dois padrões: os Estados do Maranhão, Piauí, Ceará e Rio Grande do Norte com elevados índices de Gini ao passo que os Estados da Paraíba, Pernambuco, Alagoas, Sergipe e Bahia com valores significativamente mais baixos.

A análise dos valores das elasticidades da pobreza possibilitou estabelecer uma avaliação das áreas rurais no Brasil em termos de efetividade do combate à pobreza através de políticas de crescimento e re-distributivas. Comprovou-se um resultado que está de acordo com a literatura internacional sobre o tema, a saber, que a elasticidade da 
pobreza cresce com o desenvolvimento das regiões e a distribuição de renda desigual é um entrave ao alívio da pobreza, na forma de valores mais reduzidos de elasticidade. Entretanto, deve-se levar em consideração a limitação dessa análise que não revela quantos domicílios nas regiões rurais deixariam de ser pobres.

Por outro lado, o estudo apontou que a elasticidade da pobreza se relaciona positivamente com mudanças percentuais nos índices de desigualdade. Portanto, se a pobreza pode ser reduzida mais rapidamente através da distribuição de renda, então políticas de alívio da pobreza devem ser acompanhadas por programas de redução de desigualdades de renda. É claro que a pobreza absoluta pode declinar mesmo com a presença de um nível elevado de desigualdade de renda se o crescimento da renda é suficientemente grande para compensá-lo.

Obviamente, o processo de retomada do crescimento só teria efeito sobre a redução da pobreza se ele atingisse as camadas mais pobres da população e que elas recebam os frutos do desenvolvimento econômico do país. Para isso, é necessário que a retomada do crescimento seja acompanha por programas que contemplem a geração de renda e emprego para as camadas mais pobres da população.

No caso das políticas de combate à pobreza no Nordeste elas devem considerar a importância das parcelas de renda rural, principalmente, a renda não agrícola como alternativa para reduzir o número de famílias rurais que estão abaixo da linha de pobreza e em condições de indigência.

\section{5 - Referências Bibliográficas}

BELL, C. e RICH, R. (1994) Rural poverty and agricultural performance in post - independence India, Oxford Bulletion of Economics and Statistics, 56(2), 111-133.

DATT, G. (1998) Computational tools for poverty measurement and analysis. Washington, D.C. International Food and Nutrition Institute.

DELGADO, G.; CARDOSO JR., J. C.(org.) (2000) A universalização de direitos sociais no Brasil: a previdência rural nos anos 90. Brasília, IPEA.

DUCLOS, J., ARAAR, A. E FORTIN C. (2002) DAD: Distributive analysis / Analyse Distributive, User's Manual. Université Laval. 
HELTBERG, R. (2002) The poverty elasticity of growth. Discussion Paper no. 2002/21, United Nations University/ World Institute for Development Economics research.

HOFFMAN, R. (1998) Distribuição de renda - medidas de desigualdade e pobreza. São Paulo. EDUSP.

. 2003. Desigualdade no Brasil: a contribuição das aposentadorias.

JOLLIFFE D., SEMYKINA A., (1999) Robust Standard Errors for the Foster-Greer-Thorbecke Class of Poverty Indeces: SEPOV. Stata Technical Bulletin, STB-51

JOLLIFFE, D. E BOHDAN, K., (1999) Bootstrap standard errors for indices of inequality: INEQERR, não disponível.

MOONEY, C.Z. E DUVAL, R.D, (1993) Bootstrapping: a nonparametric approach to statistical inference. Yowa. Sage Publications.

NEDER, H.D. 2001. Os efeitos das atividades não agrícolas na distribuição de renda do meio rural do Brasil. XXXIX Congresso Brasileiro de Economia e Sociologia Rural, SOBER, Anais em CD-ROM, Recife, agosto de 2001.

PESSOA, D.G.C. e SILVA, P.L.N. (1998) Análise de dados amostrais complexos. São Paulo. Associação Brasileira de Estatística.

PRATES, F. M. (1996) Desigualdade de renda e pobreza em Minas Gerais. Dissertação de Mestrado, CEDEPLAR/UFMG, Belo Horizonte.

RAVALLION, M e DATT, G. (1994) Growth and poverty in India. Washingon, DC: World Bank, Poverty and Human Resources Division (Mimeo).

SHUJIE, YAO. (1999) Economic Growth, income inequality and poverty in China under economic reforms. the Journal of Development Studies, London, vol. 35, n. 6, August, pp. 104-130

SILVA, P.L.N. (2002) An analysis of design effects for sample surveys conducted in Brazil. United nations Statistic Division.

STATA Corp., (2001) Stata Programming Guide, Release 7. College Station, Texas. Stata Press. 